\title{
Coping with COVID-19: Longitudinal Impact of the Pandemic on Adjustment and Links with Coping for Adolescents with and without ADHD
}

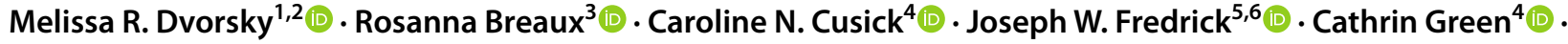

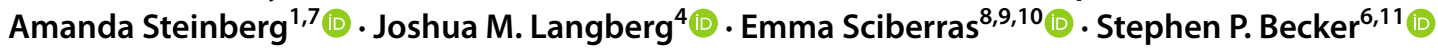

Accepted: 5 August 2021 / Published online: 7 October 2021

(c) The Author(s), under exclusive licence to Springer Science+Business Media, LLC, part of Springer Nature 2021

\begin{abstract}
Understanding factors that foster resilience and buffer against the negative psychological impact of COVID-19 is critical to inform efforts to promote adjustment, reduce risk, and improve care, particularly for adolescents with neurodevelopmental disorders. This prospective longitudinal study addresses this gap by investigating the impact of the COVID-19 pandemic on adolescents' mental health and substance use, and by assessing specific positive coping strategies among adolescents with and without attention-deficit/hyperactivity disorder (ADHD). Using multi-group autoregressive cross-lagged path models, the present study explored the reciprocal influence of positive coping behaviors on multiple adjustment outcomes including mental health symptoms, substance use, stress, and worry. Participants included 238 adolescents (132 male participants; ages 15-17; 118 with ADHD). Parents provided ratings of adolescent routines, and adolescents provided ratings of coping behaviors and psychological adjustment in spring (May/June), summer (July/August), and fall (October/November) 2020. All models included the effects of adjustment at the prior timepoint as well as relevant covariates including adolescent race, ethnicity, sex, medication status, and family income. Adolescents with ADHD were at greater risk for experiencing increases in mental health symptoms and substance use throughout the pandemic, relative to adolescents without ADHD. The use of positive coping strategies and adolescent routines buffered against increases in substance use and mental health problems for adolescents with ADHD. These findings have important clinical and public policy relevance for parents, schools, and employers who may aim to prioritize keeping schedules as consistent as possible to promote healthy adjustment.
\end{abstract}

Keywords Adolescence $\cdot$ Attention-deficit/hyperactivity disorder $\cdot$ Coping $\cdot$ Coronavirus $\cdot$ Mental health $\cdot$ Prospective

Melissa R. Dvorsky

mdvorsky@ childrensnational.org

Stephen P. Becker

Stephen.becker@cchmc.org

1 Division of Psychology and Behavioral Health, Center for Translational Research, Children's National Hospital, 111 Michigan Ave NW, Washington, DC 20010, USA

2 Department of Psychiatry \& Behavioral Sciences, and Department of Pediatrics, The George Washington University School of Medicine and Health Sciences, Washington, DC, USA

3 Department of Psychology, Virginia Polytechnic Institute and State University, Blacksburg, VA, USA

4 Department of Psychology, Virginia Commonwealth University, Richmond, VA, USA
5 Department of Psychology, Miami University, Oxford, OH, USA

6 Division of Behavioral Medicine and Clinical Psychology, Cincinnati Children's Hospital Medical Center, Cincinnati, $\mathrm{OH}$, USA

7 Department of Psychology, University of Maryland, College Park, MD, USA

8 School of Psychology, Deakin University, Geelong, Australia

9 Murdoch Children's Research Institute, Royal Children's Hospital, Melbourne, Australia

10 Department of Paediatrics, University of Melbourne, Melbourne, Australia

11 Department of Pediatrics, University of Cincinnati College of Medicine, Cincinnati, OH, USA 
Growing evidence supports the profound early impact of the COVID-19 pandemic on adolescent well-being and mental health functioning (Magson et al., 2020; Racine et al., 2020). The social disruption including interruption of school routines, stay-at-home orders, social distancing, and prolonged stress associated with COVID-19 likely intensifies adjustment difficulties in adolescents, leading to significant short- and long-term mental health concerns. Although the negative impacts of COVID-19 pose significant challenges and distress, there is considerable variability in how youth have been impacted by the pandemic (Roy et al., 2021). In addition to understanding risk and maladjustment, it is equally important to understand factors that foster resilience and buffer against the negative psychological impact of COVID-19. This is crucial to informing efforts that promote adjustment, reduce risk, and improve care particularly for adolescents both during and after the pandemic. However, prospective longitudinal studies that include multiple assessments of adjustment and mental health throughout the course of the pandemic are scarce (Breaux et al., 2021a; Magson et al., 2020). Examining mechanisms in which individuals adjust to chronic stressors like the COVID-19 pandemic has the potential to illuminate key constructs for understanding long-term coping and resilience.

\section{Psychological Impact of COVID-19 on Adolescents}

Adolescence may be a particularly difficult time for individuals to experience the disruptions associated with the COVID-19 pandemic. Adolescence is a critical developmental period associated with the prioritization of peer relationships, increased independence, as well as the onset of many mental health problems (Paus et al., 2008). However, the changes and restrictions during COVID-19 have limited independence and reduced opportunities for peer socialization, while exacerbating mental health concerns (Ren et al., 2021; Silk et al., 2021). Compared to students in elementary or middle school grades, students in high school report significantly higher levels of depression and anxiety symptoms and psychological distress in response to the COVID-19 pandemic (Tang et al., 2021). In a study of Chinese adolescents, older age and higher community infection rates predicted increased depressive symptoms during school closures in spring 2020 (Ren et al., 2021). In another study of adolescent girls using daily diaries collected during spring 2020, not having a regular schedule and problems with online schooling predicted same day increases in negative affect, depressive, and anxiety symptoms (Silk et al., 2021).

Adolescents are also likely experiencing increased worries surrounding health, safety, financial stability, and uncertainty about the future. Both cross-sectional/retrospective (Ellis et al., 2020; Tang et al., 2021) and prospective longitudinal (Magson et al., 2020) studies examining the impact of the pandemic on adolescents have highlighted the role of elevated COVID-19-related distress, anxiety, and depression symptoms. This psychological impact of the COVID-19 pandemic is likely to be magnified in adolescents with pre-existing mental health risk, such as attentiondeficit/hyperactivity disorder (ADHD).

Adolescents with ADHD appear to be particularly impacted by the coronavirus disease and its associated school closures, lockdowns, and social distancing requirements (Becker et al., 2020; Breaux et al., 2021a; Sibley et al., 2021). Adolescents with ADHD frequently experience executive functioning difficulties, co-occurring mental health concerns, emotion regulation difficulties, family conflict, academic difficulties, and substance use problems (e.g. Barkley, 2015). As such, one can imagine that adolescents with ADHD may have particular difficulty adjusting to the major changes in daily life and associated stress and uncertainty during and after the pandemic. An imminent concern for adolescents with ADHD is that pandemic-related challenges might lead to permanent negative consequences such as heavy substance use, suicidal/parasuicidal behaviors, legal problems, or school dropout (Sibley et al., 2021).

A worsening of emotional well-being and increases in mental health symptoms during COVID-19 for adolescents with ADHD has been found across cross-sectional and retrospective studies (Bobo et al., 2020; Sciberras et al., 2020; Sibley et al., 2021). In the only prospective longitudinal study of mental health symptoms from pre-COVID-19 to during the pandemic among adolescents with and without ADHD, Breaux et al. (2021a) demonstrated significant increases across adolescents in depression, anxiety, inattention, sluggish cognitive tempo, and oppositional defiant disorder symptoms from pre-COVID-19 (data collected September 2018-February 2020) to spring 2020. However, with the exception of inattention symptoms, mental health symptoms decreased from spring to summer 2020, returning on average to similar levels as pre-COVID-19.

Despite multiple calls for research on substance use problems in adolescents during the COVID-19 pandemic (e.g. Sarvey et al., 2021), very few studies have examined the impact on substance use (Dumas et al., 2020; Hawke et al., 2020) and none have examined substance use behaviors among adolescents with ADHD during COVID-19 even though adolescents with ADHD are at increased risk for substance use problems (Lee et al., 2011). A cross-sectional study of adolescents and young adults (ages 14-28) found no significant differences between retrospective report of pre-COVID-19 substance use relative to current use in April 2020 (Hawke et al., 2020). In another study of adolescents' (ages 14-18), retrospective reports of substance use (e.g. 
alcohol, cannabis, vaping) prior to the pandemic decreased on average during the pandemic (April 2020), however the number of days using alcohol and cannabis use increased (Dumas et al., 2020). Dumas and colleagues (2020) also found adolescents' substance use patterns occurring in multiple contexts during the pandemic including face-toface with friends (23.6\%), virtual contexts with friends (31.6\%), with their parents $(42.0 \%)$, and alone (49.3\%).

To date, findings on the psychological impact of COVID19 for adolescents are limited to the immediate effects assessed in the beginning of the pandemic, and less is known about the longstanding impact on adolescent mental health, COVID-related worry or stress, and substance use. Further, it is unknown whether adolescent distress or worry about COVID-19, mental health concerns, and substance use behaviors might be exacerbated by ADHD. Additionally, there is growing evidence supporting potential positive impacts since the pandemic in youth with ADHD on mental health and daily life functioning (see Breaux et al., 2021a, $2021 \mathrm{~b}$ and Dvorsky et al., 2020 for reviews). For example, adolescents with ADHD may be less likely to experience bullying (either as victims or bullies), report experiencing increased time to relax and spend time with family, as well as reduced exposure to negative feedback during remote learning and improved self-esteem (Roy et al., 2021; Sciberras et al., 2020; Sibley et al., 2021). Further, research is needed to examine individual factors that may increase or decrease risk for patterns of adjustment throughout the COVID-19 pandemic for adolescents with and without ADHD.

\section{Coping Behaviors in Adolescents and Links to Mental Health}

Although the negative impacts of COVID-19 pose significant challenges, there is considerable variability in how individuals are impacted by the pandemic. As such, it is important to understand how and why certain individuals adapt throughout and following the COVID-19 pandemic (Dvorsky et al., 2020; Prime et al., 2020). A cross-sectional study of Chinese adolescents found daily routines and physical activity protected against risks for increased depressive symptoms (Ren et al., 2021). Another study found that increased parent-child communication during the COVID-19 pandemic buffered against depressive symptoms for children and adolescents (Tang et al., 2021).

Similarly, certain individual coping factors, resources, or processes may serve as buffers against risks and promote adjustment in adolescents with ADHD (Dvorsky \& Langberg, 2016). Given the highly heterogeneous nature of ADHD, understanding factors that foster resilience is critical to informing efforts to promote adjustment, reduce risk, and improve care for individuals with ADHD both during and after the pandemic. Past research has demonstrated the importance of self-efficacy, social support, and coping behaviors for predicting resilient outcomes among adolescents with ADHD (Dvorsky et al., 2020; Dvorsky et al., 2016; Edwards et al., 2021). As COVID19 has progressed, there is emerging evidence that some youth with ADHD are functioning well in some domains, despite experiencing impairment in other domains (Sciberras et al., 2020; Sibley et al., 2021; Zhang et al., 2020).

Accumulating research highlights variability in how adolescents with ADHD are coping with the pandemic (Breaux et al., 2021b). Indeed, findings from qualitative studies highlight adolescents with ADHD report experiencing less school-related strain and reduced exposure to negative feedback (Bobo et al., 2020; Roy et al., 2021), improved self-esteem (Bobo et al., 2020; Roy et al., 2021), and more time to relax (Sibley et al., 2021). Roy and colleagues (2021) examined parent qualitative responses about specific coping strategies during COVID-19 and whether coping mechanisms differed for youth with and without ADHD. They found the most frequently endorsed strategy to support remote learning was use of routines, schedules, organization, and/or time management, with this strategy being endorsed by over half of all parents (51.4\%) but endorsed by significantly fewer parents of youth with ADHD than parents of youth without ADHD. This suggests that families of youth with ADHD may struggle more with maintaining consistent routines and schedules. Supporting this possibility, Becker et al. (2020) found that adolescent routines were negatively associated with remote learning difficulties, but only in adolescents with ADHD, suggesting routines were uniquely promotive for ADHD. Coping behaviors and routines may support positive adjustment and buffer against the negative psychological impact of COVID-19 for adolescents. Although no study has examined factors that might buffer against substance use problems in adolescents during COVID19 , one qualitative study of patients with substance use disorders found that adults who experienced coping solidarity and connectedness were more successful in abstaining from substances during the COVID-19 pandemic (DeJong et al., 2020). Finally, it remains ambiguous whether adaptive functioning/adjustment provides scaffolding for the use of coping skills during periods of stress, such as the current COVID-19 pandemic, or whether coping provides precedes and therefore contextualizes adjustment and socio-emotional development. The current study builds upon prior work by examining multiple areas of COVID-19 adjustment among 
adolescents with and without ADHD and examining potential reciprocal and transactional patterns between adjustment and coping from spring to fall 2020.

\section{Present Study}

The present study sought to use a prospective, longitudinal study of adolescents to examine changes in, and predictors of, adjustment during the COVID-19 pandemic in adolescents with and without ADHD. We considered the influence of coping behaviors on multiple adjustment outcomes including mental health symptoms, substance use, and COVIDrelated stress and worry. The first aim of this study was to examine changes in mental health symptoms, substance use, COVID-related stress, COVID-related worry, and coping behaviors in adolescents with and without ADHD. Based on extant research (Breaux et al., 2021a; Roy et al., 2021), it was hypothesized that adolescents with ADHD would experience more adjustment difficulties and be less likely to engage in coping strategies, relative to adolescents without ADHD. The second aim was to examine whether the reciprocal influence of changes in adjustment and coping during the pandemic differed for adolescents with and without ADHD. To address this aim, we use cross-lagged path analyses on three waves of data (from spring 2020 to late fall 2020) to understand the temporal sequence (i.e. multiple reciprocal processes) linking coping and adjustment. We hypothesized a negative, prospective relation between coping and adjustment difficulties, specifically mental health symptoms, substance use, COVID-19 related stress, and COVID-19 worry. Additionally, we explored whether the reverse direction was true; that is, whether adjustment predicted subsequent coping behaviors. The inclusion of ADHD status enabled us to examine whether bidirectional associations differ between adolescents with and without ADHD. Given research on active coping strategies buffering against COVID-19 related stress (Ren et al., 2021; Tang et al., 2021), we hypothesized that adolescents who engage in more coping behaviors will experience fewer mental health symptoms, less substance use, less COVID-related stress, and less worry about the pandemic. Further, based on findings that adolescents with ADHD and poorer emotional regulation abilities display the highest symptomatology throughout the pandemic (Breaux et al., 2021a; McFayden et al., 2021), we hypothesized the association between coping behaviors and adjustment would be most pronounced for youth with ADHD. Finally, as an exploratory aim, we examined multi-group analyses examining each domain of coping behavior (i.e. self-care behaviors, solitary activities, social behaviors, technology, creative expression) as separate predictors in each of the adjustment outcomes, and whether associations differed for adolescents with/without ADHD.

\section{Methods}

\section{Participants and Procedures}

Participants were 238 adolescents (132 were male participants; ages 15-17 years) with and without ADHD who were initially recruited in eighth grade from local public schools across Ohio, Kentucky, and Virginia (United States). Based on a comprehensive diagnosis at the initial evaluation prior to COVID-19, 118 of the adolescents were diagnosed with ADHD (85 ADHD predominantly inattentive presentation, 33 ADHD combined presentation), with the remaining participants $(n=120)$ comprising a comparison sample without ADHD. Adolescents identified as predominantly White (82\%), with $7 \%$ identifying as biracial/multiracial, $6 \%$ identifying as Black, $4 \%$ Asian, and $1 \%$ identifying as another race; $4 \%$ of the sample identified as Latinx. Participants came from a range of socioeconomic backgrounds ( $M$ family income $=\$ 93,073, S D=\$ 34,856$ ), with $19 \%$ of families falling below the 2019 United States median household income. Details of the parent study and diagnostic procedures can be found in the Supplemental Materials, and further description of the sample are provided in Table S1.

Participants from the larger study (Becker et al., 2019; Langberg et al., 2019) who provided permission for further contact pre-COVID-19 (visits between September 2018 and February 2020; $N=262 ; 90.8 \%$ retention) were invited to participate in the current study, with COVID-19 data being collected between May 15 and June 14, 2020 during initial stay-at-home orders (spring 2020), between July 1 and August 5, 2020 (summer 2020), and between October 15 and November 19, 2020 (fall 2020). The 238 participants who participated in the COVID-19 timepoints did not differ from 24 participants who were contacted for possible participation on adolescent sex $(d=0.13)$, race $(d=0.16)$, ethnicity $(d=0.09)$, family income $(d=0.13)$, ADHD symptoms $(d=0.31)$, overall impairment $(d=0.30)$, or self-reported internalizing symptoms $(d=0.17)$, all $p s>0.07$. Parents and adolescents provided consent and assent, respectively, for participation and were compensated for participation at all timepoints. Data at all timepoints was collected online via REDCap. This study was approved by the Cincinnati Children's Hospital Medical Center and Virginia Commonwealth University Institutional Review Boards.

\section{Measures}

\section{Coronavirus Health Impact Survey (CRISIS)}

The CRISIS (Nikolaidis et al., 2020) was developed by National Institute of Mental Health (NIMH) investigators 
and collaborators (www.crisissurvey.org) and was completed by adolescents at each of the three COVID-19 timepoints. To measure the impact of COVID-19 on adjustment in the present study, items from the adolescent version assessing mental health symptoms, substance use, worry about COVID-19, and stress related to COVID-19 restrictions/changes were utilized. The worry scale consists of five items assessing worries about COVID-19 (e.g. "How worried have you been about being infected?", "How worried have you been about your physical health being influenced by COVID-19?") rated on a five-point scale $(1=$ not at all/none $; 5=$ extremely/a great amount $)$. Mean scores were calculated to create a worry composite, with higher scores indicating high levels of worry related to the pandemic ( $\alpha s=0.82-0.84)$. Five items additional assess stress associated with COVID-19 restrictions/changes (e.g. "How stressful have the restrictions on leaving home been for you?", "How stressful have these changes in social contacts been for you?") rated on a five-point scale ( $1=n o t$ at all/none; $5=$ extremely/a great amount). A mean composite score for COVID-related stress was calculated, with higher scores indicating high levels of stress from the pandemic ( $\alpha s=0.70-0.76)$. The mental health scale consists of 10 items rated on a five-point scale $(1=$ not at all; $5=$ extremely) assessing adolescents' emotions/feelings associated with their mental health (e.g. sadness, restlessness, anxiety, fatigue) over the past two weeks. Mean scores were calculated to create a mental health composite, with higher scores indicating greater mental health symptom severity ( $\alpha \mathrm{s}=0.86-0.88$ ). Three items assessing the frequency of substance use including alcohol, vaping, and cigarettes were rated on a five-point scale $(1=$ not at all; $5=$ regularly), with higher scores indicating higher frequency of use ( $\alpha s=0.66-0.73)$. In the present study, all CRISIS items assessed functioning during the past two weeks and were assessed at all three timepoints during COVID-19, with the exception of the substance use scale which was assessed retrospectively in the three months prior to COVID-19 during spring 2020, and at the spring 2020 and summer 2020 COVID-19 timepoints (but not in fall 2020).

\section{COVID-19 Adolescent Symptom and Psychological Experience Questionnaire (CASPE)}

The CASPE (Ladouceur, 2020) is an adolescent-report measure designed to assess experiences related to COVID19 including various coping strategies and was completed by adolescents at all three COVID-19 timepoints. Five mean composites were created: self-care behaviors (4 items; e.g. getting a good night's sleep, exercising, eating healthier), solitary behaviors ( 4 items; e.g. reading a book, meditation practice), social behaviors (4 items; e.g. talking with friends, playing board games, engaging in more family activities), technology (4 items; e.g. playing video games, watching a movie), and creative expression (3 items; e.g. arts or crafts, writing). Each coping strategy item is rated either yes (1) or no (0) and items on each scale are averaged such that higher mean scores indicate higher levels of engagement in coping behaviors. A global mean composite of all coping behaviors was utilized in the autoregressive cross-lagged models in the present study $(\alpha \mathrm{s}=0.73-0.77)$.

\section{Adolescent Routines Questionnaire (ARQ)}

Parents completed the ARQ (Piscitello et al., 2019) to assess adolescents' engagement in daily routines, activities, and structure as a potential coping mechanism during the pandemic. Items are rated on a five-point scale ranging from $0=$ Never to $4=$ Nearly Always. A total composite score across 26 items was used in the present study to assess adolescent daily routines ( $\alpha s=0.89-0.91$ ). The ARQ has demonstrated acceptable internal consistency $(\alpha s=0.82-0.85)$ and construct validity in a sample of adolescents 12-17 (Piscitello et al., 2019). Specifically, adolescent routines as measured by the ARQ parent-report is positively correlated with parent-report of family routines $(r=0.41)$ and adolescent adaptive skills $(r=0.58)$, and negatively associated with executive functioning skills deficits $(r=0.56)$ (Piscitello et al., 2019).

\section{Demographic Variables}

Adolescents reported on their racial $($ Black $=1$, nonBlack $=0)$ and ethnic (Latinx $=1$, non-Latinx $=0)$ identity. Parents reported adolescents' biological sex (male $=0$; female $=1$ ) and family income all timepoints. Three medication status covariates were used based on parent-report of medication on the Vanderbilt ADHD Diagnostic Rating Scale (Wolraich et al., 2003) at each timepoint $(1=$ on medication, $0=$ not on medication or unsure).

\section{Analyses}

Descriptive statistics were examined to assess differences in coping behaviors, mental health symptoms, substance use, COVID-19 related stress, and worries about COVID-19 for adolescents with and without ADHD throughout the pandemic. The bidirectional and reciprocal influences between coping, routines, and adjustment were examined using multigroup autoregressive cross-lagged models (ARCL) path analysis with MPlus 8.4. All models used the maximum likelihood estimation with robust standard errors (MLR), which computes mean-adjusted maximum likelihood estimates for nonnormally distributed data and is recommended for small and medium sample sizes (Muthén \& Muthén, 2020). 
Chi-square goodness-of-fit indices and incremental fit indices including the comparative fit index (CFI; values $>0.90$ ), and root-mean-square error of approximation (RMSEA; values $<0.08$ ) were examined for adequate model fit (Wang \& Wang, 2012).

Each model included the autoregressive paths (predicting a construct from its prior level) and the cross-lagged paths (connecting coping behaviors and adjustment across adjacent time points). Three ARCL models were fit separately for (a) worries about COVID, (b) COVID-related stress, and (c) mental health symptoms, with adolescent coping behavior and routines across the three study waves. A fourth model was fit for substance use from the retrospective report of preCOVID-19 substance use and across two timepoints during COVID-19 (spring and summer 2020) with the same coping and routines variables included. Adolescents with ADHD were more likely male adolescents $(d=0.40)$ and to take medication for ADHD ( $d \mathrm{~s}=0.57-1.09$; see Table $\mathrm{S} 1)$. As such, all models included adolescent sex, race, ethnicity, cohort, and medication status at each of the three timepoints as covariates. Residual correlations at each wave were also specified to examine the concurrent correlations between coping, routines, and adjustment, unexplained by the cross-lagged associations. After a model was established that fit the data, we tested whether the stability paths could be constrained to be equal over time. Next, we tested whether the cross-lagged paths between coping/routines and the adjustment outcome could be constrained to be equal over time. Satorra-Bentler scaled $\chi^{2}$ difference tests (Satorra \& Bentler, 2001) were used to compare the fit of nested models. ${ }^{1}$ If constraining paths to be equal over time did not result in a significant decrease in model fit, this indicates that there is time-invariance. Group differences in significant cross-lagged paths were examined with Wald Tests. Finally, exploratory multi-group path models examined the relative influence of each domain of coping behaviors (self-care, solitude, social behavior, technology, creative expression) and routines assessed in the spring 2020 for predicting adolescent COVID-related worry, stress, mental health symptoms, and substance use in the summer 2020, controlling for initial levels.

\section{Results}

\section{Preliminary Analyses}

Descriptive statistics for the total sample, ADHD group, and non-ADHD comparison group are presented in Table 1.

\footnotetext{
1 To conserve space, model fit statistics are only reported for final models. Fit statistics and results of the chi-square difference tests are available on request of the first author.
}

Adolescents with ADHD engaged in significantly less routines $(d \mathrm{~s}=0.75-0.92)$ and coping strategies $(d s=0.32-0.39)$ at all timepoints during COVID-19, compared to those without ADHD. At the same time, adolescents without ADHD demonstrated greater worry about COVID-19 $(d s=0.21-0.51)$ in the spring and summer, relative to adolescents with ADHD. Adolescents with ADHD reported higher levels of substance use approaching significance in spring $2020(d=0.22)$ and significant during summer 2020 $(d=0.35)$ relative to adolescents without ADHD, despite similar mean levels on retrospective report of substance use three months prior to COVID (ADHD: $M=3.19, S D=0.76$; without ADHD: $M=3.33, S D=1.19 ; t(260)=1.04$, $p=0.303, d=0.13)$. Initially, there were no significant differences in mental health symptoms in the spring; however, in the summer and fall adolescents with ADHD reported higher levels of mental health symptoms $(d s=0.26-0.29)$. Overall, adolescents without ADHD reported higher levels of stress from COVID-19 relative to adolescents with ADHD, although this was only significantly different in the summer $(d=0.25)$.

Bivariate correlations among all of the coping behaviors, routines, and adjustment outcomes across all three time points are presented in Table S2. Coping and routines were generally associated with less mental health symptoms $(r \mathrm{~s}=0.00$ to -0.24$)$ and lower levels of substance use ( $r \mathrm{~s}=-0.07$ to -0.37 ). Worries about COVID and COVIDrelated stress negatively correlated with adolescent routines, but positively correlated with coping behaviors. COVIDrelated stress and worry were modestly correlated across time ( $r \mathrm{~s}=0.22$ to 0.44 ), suggesting these constructs were not redundant, representing unique aspects of adjustment to COVID-19.

\section{Cross-Lagged Models of Coping Behaviors, Routines, and Adjustment}

For all models, constraining the stability paths resulted in a significant decrease in model fit, indicating that the stability of coping, routines, and adjustment changed over time. Similarly, for all models, constraining the cross-lagged paths resulted in a significant decrease in model fit, indicating these associations varied over time. As such for each outcome, stability and cross-lagged paths were freely estimated. Standardized parameters are presented in Figs. 1 and 2.

\section{Mental Health Symptoms}

The model examining the longitudinal association and reciprocal effects of adolescent routines, coping, and mental health symptoms (Fig. 1a) demonstrated good model fit $\left(C F I=0.96, R M S E A=0.06, \chi^{2}(95)=134.30\right.$, $p=0.01)$. For adolescents with ADHD, adolescent 
Table 1 Descriptive Statistics of Coping and Adjustment for Total, ADHD, and Comparison Samples

\begin{tabular}{|c|c|c|c|c|c|}
\hline & $\begin{array}{l}\text { Total Sample } \\
M(S D) \\
N=238\end{array}$ & $\begin{array}{l}\text { ADHD Sample } M(S D) \\
N=118\end{array}$ & $\begin{array}{l}\text { Comparison Sample } \\
M(S D) \\
N=120\end{array}$ & $t$ & $d$ \\
\hline & \multicolumn{5}{|l|}{ Spring 2020} \\
\hline Worries About COVID-19 & $2.29(.75)$ & $2.17(.75)$ & $2.40(.74)$ & $2.37 * *$ & .31 \\
\hline Stress from COVID-19 & $2.36(.83)$ & $2.32(.89)$ & $2.40(.76)$ & .70 & .10 \\
\hline Impact of COVID-19 on Mental Health & $2.62(.74)$ & $2.65(.75)$ & $2.60(.74)$ & -.48 & .06 \\
\hline Impact of COVID-19 on Substance Use & $4.91(1.55)$ & $5.08(1.34)$ & $4.73(1.73)$ & $-1.70^{\dagger}$ & .22 \\
\hline Parent Rated Adolescent Routines & $46.16(12.32)$ & $41.46(12.51)$ & $50.75(10.27)$ & $6.22 * * *$ & .82 \\
\hline Coping Strategies (Mean) & $1.45(.66)$ & $1.32(.66)$ & $1.57(.64)$ & $2.97 * *$ & .39 \\
\hline Coping with Self-Care & $1.53(1.24)$ & $1.25(1.11)$ & $1.81(1.30)$ & $3.50 * *$ & .46 \\
\hline Coping with Solitude & $1.04(.91)$ & $1.01(.94)$ & $1.08(.88)$ & .58 & .08 \\
\hline Coping with Social Behavior & $1.49(1.05)$ & $1.41(1.05)$ & $1.57(1.04)$ & 1.17 & .15 \\
\hline Coping with Technology & $2.59(1.11)$ & $2.49(1.26)$ & $2.69(.93)$ & 1.39 & .18 \\
\hline \multirow[t]{2}{*}{ Coping with Creative Expression } & $.57(.79)$ & $.44(.70)$ & $.71(.85)$ & $2.64 * *$ & .35 \\
\hline & \multicolumn{5}{|l|}{ Summer 2020} \\
\hline Worries About COVID-19 & $2.08(.75)$ & $1.88(.71)$ & $2.25(.75)$ & $3.87 * * *$ & .51 \\
\hline Stress from COVID-19 & $2.09(.72)$ & $1.99(.76)$ & $2.18(.66)$ & $1.86^{*}$ & .25 \\
\hline Impact of COVID-19 on Mental Health & $2.40(.66)$ & $2.48(.71)$ & $2.30(.61)$ & $-1.95^{*}$ & .26 \\
\hline Impact of COVID-19 on Substance Use & $4.85(1.54)$ & $5.13(1.47)$ & $4.59(1.57)$ & $-2.67 * *$ & .35 \\
\hline Parent Rated Adolescent Routines & $47.37(11.14)$ & $43.49(11.86)$ & $51.22(8.87)$ & $5.64 * * *$ & .75 \\
\hline Coping Strategies (Mean) & $1.47(.65)$ & $1.36(.66)$ & $1.58(.63)$ & $2.54 * *$ & .34 \\
\hline Coping with Self-Care & $1.68(1.16)$ & $1.52(1.08)$ & $1.84(1.22)$ & $2.07 *$ & .27 \\
\hline Coping with Solitude & $1.12(.97)$ & $1.07(.96)$ & $1.16(.98)$ & .70 & .09 \\
\hline Coping with Social Behavior & $1.41(1.05)$ & $1.30(1.08)$ & $1.52(1.01)$ & $1.62^{\dagger}$ & .22 \\
\hline Coping with Technology & $2.61(1.10)$ & $2.49(1.21)$ & $2.74(.97)$ & $1.72^{\dagger}$ & .23 \\
\hline \multirow[t]{2}{*}{ Coping with Creative Expression } & $.53(.75)$ & $.42(.67)$ & $.63(.81)$ & $2.23 *$ & .30 \\
\hline & \multicolumn{5}{|l|}{ Fall 2020} \\
\hline Worries About COVID-19 & $2.07(.83)$ & $1.94(.85)$ & $2.18(.80)$ & 1.37 & .18 \\
\hline Stress from COVID-19 & $2.06(.72)$ & $2.05(.81)$ & $2.07(.63)$ & .17 & .03 \\
\hline Impact of COVID-19 on Mental Health & $2.53(.52)$ & $2.59(.55)$ & $2.49(.48)$ & $2.14^{*}$ & .29 \\
\hline Parent Rated Adolescent Routines & $68.24(15.49)$ & $61.23(16.81)$ & $74.71(10.70)$ & $7.23 * * *$ & .97 \\
\hline Coping Strategies (Mean) & $1.27(.66)$ & $1.16(.69)$ & $1.37(.62)$ & $2.38 *$ & .32 \\
\hline Coping with Self-Care & $1.39(1.21)$ & $1.12(1.18)$ & $1.63(1.19)$ & $3.25 * * *$ & .44 \\
\hline Coping with Solitude & $0.95(.87)$ & $0.84(.81)$ & $1.04(.90)$ & $1.78^{\dagger}$ & .24 \\
\hline Coping with Social Behavior & $1.14(.94)$ & $1.14(.98)$ & $1.14(.91)$ & -.04 & .01 \\
\hline Coping with Technology & $2.37(1.23)$ & $2.29(1.38)$ & $2.44(1.09)$ & .93 & .13 \\
\hline Coping with Creative Expression & .48 & $.39(.70)$ & $.57(.79)$ & $1.70^{\dagger}$ & .23 \\
\hline
\end{tabular}

$d f=236$ at Spring and Summer comparisons and $d f=225$ for fall comparisons. Cohen's $d$ was calculated as a measure of effect size, with $.3, .5$, and .8 representing small, medium, and large effects, respectively (Cohen, 1988). ${ }^{\dagger} p<.10,{ }^{*} p<.05, * * p<.01 * * * p<.001$

routines and coping in the spring predicted decreased mental health symptoms in summer, and this pattern persisted for coping behaviors in the fall. For adolescents without ADHD, coping behaviors in the summer approached significance in predicting mental health in the fall $(\beta=0.16, p=0.072)$, and other cross-lagged effects were nonsignificant. Moreover, for adolescents with ADHD, increased mental health symptoms in the spring was associated with greater coping behaviors in the summer, although this effect was not significant in the fall and not observed for adolescents without ADHD. Moreover, Wald tests showed that these cross-lagged paths were significantly stronger for adolescents with ADHD than adolescents without ADHD (Wald $=22.54$, $p=0.004$ ). Adolescent sex was the only covariate significantly associated with mental health symptoms such that 
female adolescents had greater symptom severity across timepoints $(p s<0.001){ }^{2}$

\section{Worries About COVID-19}

The model for COVID-19-related worries (Fig. 1b) demonstrated adequate model fit $(C F I=0.98, R M S E A=0.04$, $\left.\chi^{2}(95)=112.67, p=0.103\right)$. For adolescents with ADHD, increased worry about COVID-19 in spring was associated with increased coping behaviors and routines in the summer, and in turn increased coping in the summer was associated with decreased worry in the fall. None of the cross-lagged paths were significant between coping and worries about COVID-19 for adolescents without ADHD, yet Wald tests found cross-lagged paths between coping, routines, and worry did not significantly vary in magnitude between adolescents with and without ADHD (Wald $=14.52, p=0.069$ ).

\section{COVID-19 Related Stress}

For the model examining COVID-related stress (Fig. 1c; $C F I=0.98, R M S E A=0.04, \chi^{2}(95)=112.05, p=0.100$ ) increased initial stress related to COVID-19 in spring and summer was associated with increased coping behaviors and in the summer and subsequent fall, only for adolescents with ADHD. Increased use of routines in the summer predicted lower levels of stress about COVID-19 in the fall, again only for adolescents with ADHD but not those without ADHD. Moreover, Wald tests showed that these cross-lagged paths were significantly stronger for adolescents with ADHD than adolescents without ADHD (Wald $=17.30, p=0.027$ ).

\footnotetext{
$\overline{2}$ Our primary aim was to examine ADHD as a potential moderator of the relations between coping/routines and adjustment outcomes during the COVID-19 pandemic. Since tests of statistical interactions are typically underpowered in nonexperimental research (McClelland \& Judd, 1993), we chose to limit the number of covariate or predictor variables included in these models. Further, while including all important covariates and predictor variables and examining multiple outcomes of adjustment strengthens confidence in the findings, increasing the number of variables and paths to be tested, also increases the probability of a Type 1 error. We viewed the inclusion parent- and adolescent-rated coping factors in the path models predicting adjustment as important to expanding understanding of the longitudinal and potentially reciprocal associations over time to inform future research. Nonetheless, we acknowledge that the decision to examine each of these outcome domains separately may have increased the probability of having a Type 1 error. There is no simple solution to this dilemma, particularly as a Bonferroni or related corrections have been heavily criticized for creating additional methodological and statistical problems (Nagagawa, 2004; Moran, 2003; Perneger, 1998). Therefore, we did not apply such a correction, but acknowledge that if such a correction were used the effects of a) T1 stress to T2 coping; b) T1 worry to T2 routines; and c) T1 coping to $\mathrm{T} 2$ mental health symptoms and T2 substance use would be lost.
}

Adolescent sex was the only covariate significantly associated with stress such that female adolescents were associated with greater levels of stress across time $(p s<0.001)$.

\section{Substance Use}

In the model examining substance use (Fig. 2; $C F I=0.91$, $\left.R M S E A=0.08, \chi^{2}(110)=194.01, p<0.001\right)$, adolescent routines in the spring significantly predicted decreased substance use in the summer for both adolescents with and without ADHD. Coping behaviors in the spring predicted decreased substance use in the summer, but only for adolescents with ADHD. Increased substance use in the summer was associated with less use of routines and coping in the fall, only for adolescents with ADHD. Cross-lagged paths were significantly stronger for adolescents with ADHD compared to adolescents without ADHD (Wald $=18.27, p=0.006$ ).

\section{Path Models of Routines and Individual Coping Behaviors Predicting Adjustment}

Based on the observed longitudinal associations and reciprocal effects between coping behaviors and mental health symptoms, substance use, worry, and stress in the ARCL models, we then conducted additional exploratory multigroup path models predicting adjustment using the individual coping subscales (see Table 2). For mental health symptoms, controlling for spring levels of mental health severity, solitary coping $(\beta=-0.012, p=0.040)$, social coping $(\beta=-0.01$, $p=0.038)$, coping with technology $(\beta=-0.02, p=0.010)$, and adolescent routines $(\beta=-0.16, p=0.002)$ significantly predicted decreased mental health symptoms in the summer for adolescents with ADHD. None of the coping factors predicted mental health severity for adolescents without ADHD. Race was significantly associated with summer mental health severity $(\beta=-0.18, p=0.010)$ such that for adolescents without ADHD, those who identified as Black were associated with greater severity of symptoms. Overall, these variables predicted $58 \%$ of the variance in mental health severity for adolescents with ADHD and 55\% of the variance for adolescents without ADHD. For predicting substance use, controlling for initial levels of substance use in the spring, coping with technology $(\beta=-0.14, p=0.033)$, and adolescent routines ( $\beta=-0.15, p=0.027$ ) significantly predicted decreased substance use in the summer for adolescents with ADHD. Overall, these variables predicted $60 \%$ of the variance in substance use for adolescents with ADHD and $49 \%$ of the variance for adolescents without ADHD. For worry related to COVID-19, controlling for initial levels of worry in the spring, self-care $(\beta=-0.02, p=0.046)$, solitary coping $(\beta=-0.01, p=0.049)$, social coping $(\beta=-0.01$, $p=0.007)$, and adolescent routines $(\beta=-0.11, p=0.041)$ significantly predicted worry in the summer for adolescents 
Table 2 Multi-Group

Moderated Regression

Models of Adolescent Coping

Predicting Adjustment

\begin{tabular}{|c|c|c|c|c|c|c|}
\hline \multirow[t]{2}{*}{ Spring 2020 Predictors } & \multicolumn{3}{|c|}{ ADHD Sample } & \multicolumn{3}{|c|}{ Comparison Sample } \\
\hline & $\beta$ & $S E$ & $t$ & $B$ & $S E$ & $t$ \\
\hline & \multicolumn{6}{|c|}{$\begin{array}{l}\text { DV: Adolescent COVID Impact on Mental Health (Summer } \\
\text { 2020) }\end{array}$} \\
\hline Initial Mental Health Symptoms & .62 & .08 & $8.19 * * *$ & .58 & .06 & $10.59 * * *$ \\
\hline Cohort & .15 & .09 & 1.65 & .09 & .07 & 1.26 \\
\hline Black & .08 & .10 & .82 & -.18 & .07 & $-2.53 *$ \\
\hline Latinx & .04 & .09 & .44 & .07 & .05 & 1.33 \\
\hline Sex & .02 & .11 & .20 & .11 & .07 & 1.56 \\
\hline Medication Status & .05 & .08 & .64 & .08 & .07 & 1.24 \\
\hline Parent Rated Adolescent Routines & -.16 & .06 & $-2.56 * *$ & -.06 & .06 & -.97 \\
\hline Coping with Self-Care Behaviors & -.01 & .01 & $-1.80^{\dagger}$ & -.01 & .01 & -.95 \\
\hline Coping with Solitude & -.01 & .01 & $-2.14 *$ & -.01 & .01 & -.94 \\
\hline Coping with Social Behaviors & -.01 & .01 & $-2.05^{*}$ & -.01 & .01 & -.95 \\
\hline Coping with Technology & -.02 & .01 & $-2.69 * *$ & -.01 & .01 & -.95 \\
\hline Coping with Creative Expression & -.01 & .01 & $-1.73^{\dagger}$ & -.01 & .01 & -.95 \\
\hline
\end{tabular}

DV: Adolescent COVID Impact on Substance Use (Summer 2020)

\begin{tabular}{|c|c|c|c|c|c|c|}
\hline Initial Substance Use & .60 & .09 & $6.52 * * *$ & .67 & .10 & $6.58 * * *$ \\
\hline Cohort & .06 & .07 & .88 & -.04 & .06 & -.68 \\
\hline Black & -.03 & .09 & -.34 & -.03 & .05 & -.62 \\
\hline Latinx & .04 & .12 & .34 & -.06 & .05 & -1.36 \\
\hline Sex & -.07 & .09 & -.81 & -.12 & .10 & -1.18 \\
\hline Medication Status & .02 & .04 & .41 & .14 & .10 & 1.44 \\
\hline Parent Rated Adolescent Routines & -.15 & .07 & $-2.00 *$ & -.05 & .08 & -.60 \\
\hline Coping with Self-Care Behaviors & -.02 & .01 & -1.32 & -.01 & .01 & -.60 \\
\hline Coping with Solitude & -.12 & .01 & $-1.61^{\dagger}$ & -.00 & .01 & -.59 \\
\hline Coping with Social Behaviors & -.11 & .01 & $-1.60^{\dagger}$ & -.01 & .01 & -.60 \\
\hline Coping with Technology & -.14 & .01 & $-2.03 *$ & -.00 & .01 & -.59 \\
\hline \multirow[t]{2}{*}{ Coping with Creative Expression } & -.01 & .01 & -1.29 & -.00 & .01 & -.60 \\
\hline & \multicolumn{6}{|c|}{ DV: Adolescent COVID-Related Worries (Summer 2020) } \\
\hline Initial COVID-Related Worries & .48 & .08 & $6.08 * * *$ & .61 & .06 & $11.16^{* * *}$ \\
\hline Cohort & .11 & .09 & 1.22 & -.09 & .07 & -1.36 \\
\hline Black & -.03 & .12 & -.28 & -.13 & .09 & $-1.88^{\dagger}$ \\
\hline Latinx & -.02 & .18 & -.11 & -.07 & .08 & -.83 \\
\hline Sex & .17 & .10 & 1.73 & -.04 & .08 & -.57 \\
\hline Medication Status & -.13 & .11 & -1.19 & -.04 & .07 & -.52 \\
\hline Parent Rated Adolescent Routines & -.01 & .07 & -.18 & -.11 & .05 & $-1.97 *$ \\
\hline Coping with Self-Care Behaviors & .00 & .01 & .13 & -.02 & .01 & $-1.96^{*}$ \\
\hline Coping with Solitude & .00 & .01 & .15 & -.01 & .01 & $-1.94 *$ \\
\hline Coping with Social Behaviors & .00 & .01 & .15 & -.01 & .01 & $-1.95^{*}$ \\
\hline Coping with Technology & .00 & .01 & .19 & -.01 & .01 & $1.91^{\dagger}$ \\
\hline \multirow[t]{2}{*}{ Coping with Creative Expression } & .00 & .01 & .12 & -.01 & .01 & $1.91^{\dagger}$ \\
\hline & \multicolumn{6}{|c|}{ DV: Adolescent COVID-Related Stress (Summer 2020) } \\
\hline Initial COVID-Related Stress & .55 & .08 & $6.78 * * *$ & .56 & .08 & $7.16^{* * * *}$ \\
\hline Cohort & .09 & .10 & .96 & .03 & .07 & .39 \\
\hline Black & .14 & .11 & 1.23 & .05 & .07 & .80 \\
\hline Latinx & .09 & .12 & .76 & .06 & .11 & .58 \\
\hline Sex & .10 & .10 & .98 & .00 & .10 & .02 \\
\hline Medication Status & -.03 & .07 & -.43 & .07 & .11 & .70 \\
\hline Parent Rated Adolescent Routines & -.04 & .06 & -.59 & .02 & .09 & -.01 \\
\hline Coping with Self-Care Behaviors & -.00 & .01 & -.42 & .00 & .01 & -.01 \\
\hline
\end{tabular}


Table 2 (continued)

\begin{tabular}{|c|c|c|c|c|c|c|c|}
\hline \multirow[t]{2}{*}{ Spring 2020 Predictors } & \multicolumn{4}{|c|}{ ADHD Sample } & \multicolumn{3}{|c|}{ Comparison Sample } \\
\hline & $\beta$ & $S E$ & & $t$ & $B$ & $S E$ & $t$ \\
\hline Coping with Solitude & -.00 & .01 & -.50 & & .00 & .01 & -.01 \\
\hline Coping with Social Behaviors & -.00 & .01 & -.48 & & .00 & .01 & -.01 \\
\hline Coping with Technology & -.00 & .01 & -.63 & & .00 & .01 & -.01 \\
\hline Coping with Creative Expression & -.00 & .01 & -.40 & & .00 & .01 & -.01 \\
\hline
\end{tabular}

Medication status $(0=$ not medicated, $1=$ on medication $) .^{\dagger} p<.10, * p<.05, * * p<.01 * * * p<.001$

without ADHD. Overall, these variables predicted $30 \%$ of the variance in worries about COVID-19 for adolescents with ADHD and $47 \%$ of the variance for adolescents without ADHD. None of the coping behaviors or routines predictors were significant in the model predicting COVID-related stress for adolescents with or without ADHD.

\section{Discussion}

This study used a prospective longitudinal design to examine changes in adjustment to the COVID-19 pandemic across mental health symptoms, stress, worry, and substance use among adolescents with and without ADHD using data collected during initial stay-at-home orders in the spring
Fig. 1 Cross-lagged Path Model Examining Bidirectional Effects Between Coping and Adjustment. Standardized parameter estimates are reported. Non-significant and covariate parameters remain in the model but not are not displayed in the figure. Path coefficients without parentheses are for ADHD sample; path coefficients in parentheses are for non-ADHD comparison sample. ${ }^{\dagger} p<.10$ $* p<.05 . * * p<.01 . * * * p<.001$ a) Mental Health Symptoms

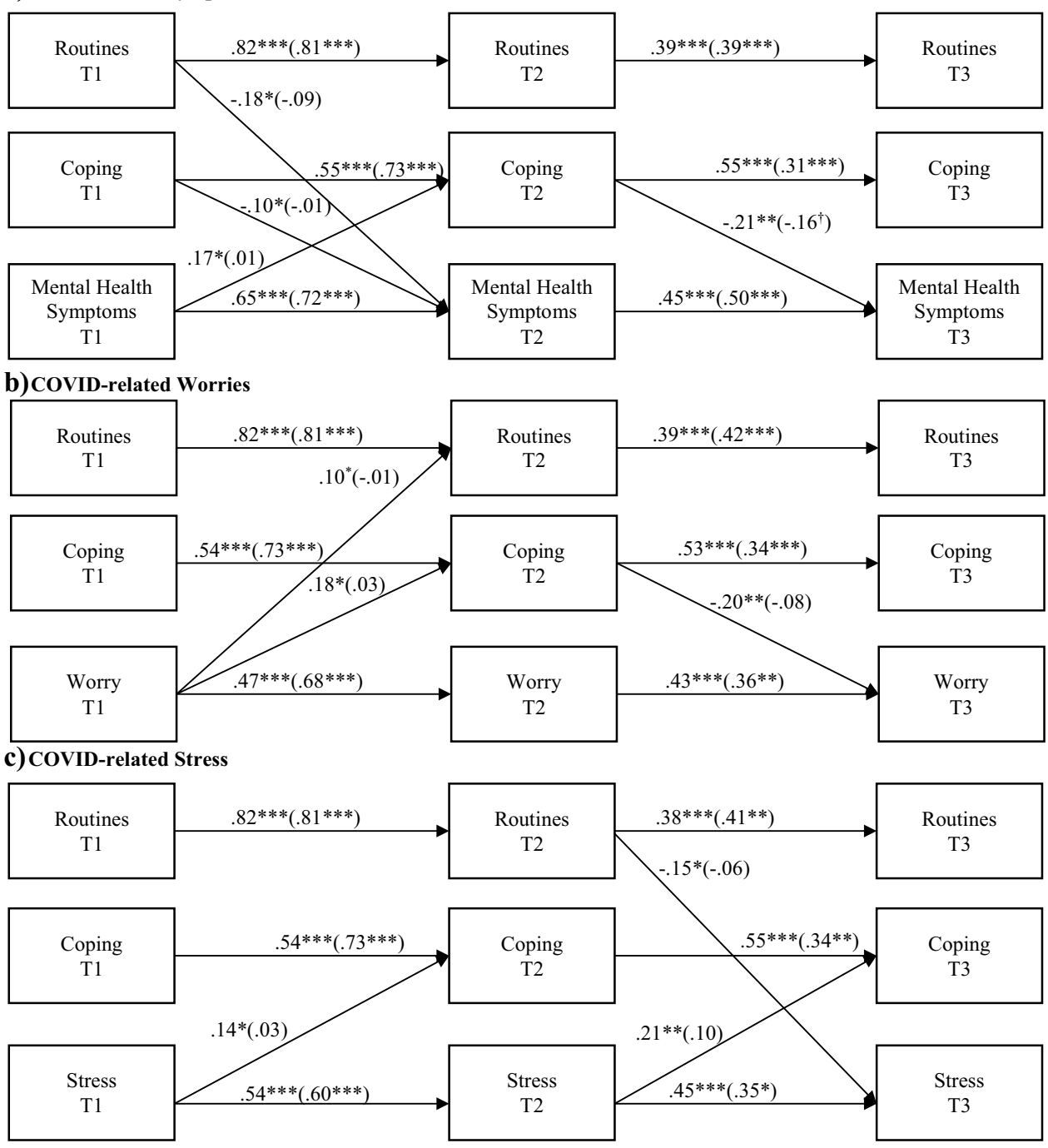

Note. Standardized parameter estimates are reported. Non-significant and covariate parameters remain in the model but not are not displayed in the figure. Path coefficients without parentheses are for ADHD sample; path coefficients in parentheses are for non-ADHD comparison sample. ${ }^{\dagger} p<.10 * p<.05 . * * p<.01 .{ }^{* * *} p<.001$. 


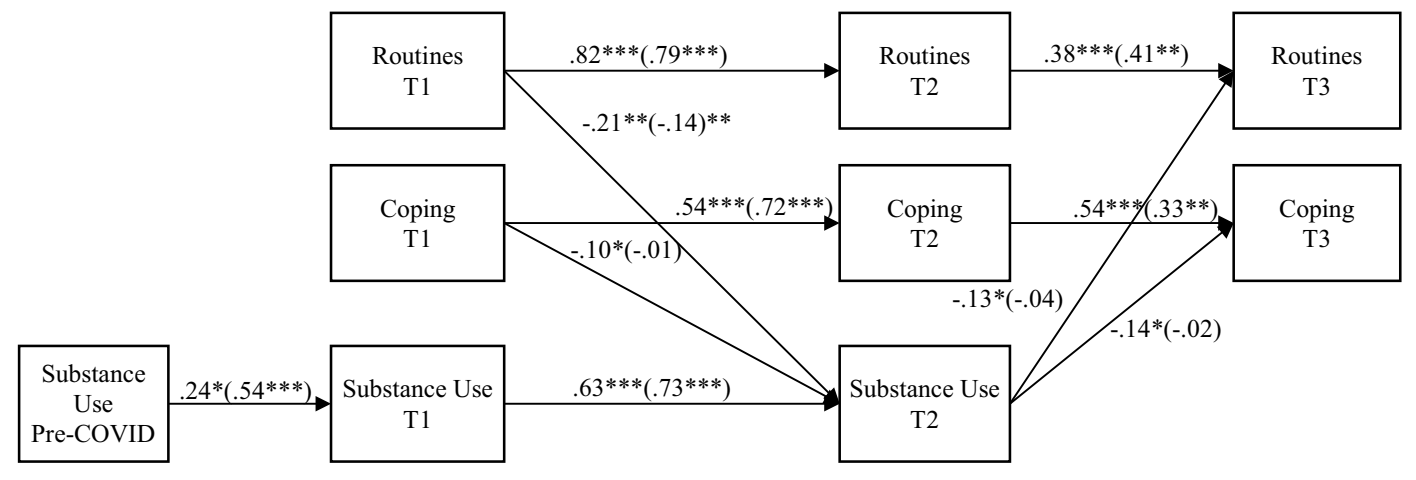

Note. Standardized parameter estimates are reported. Non-significant and covariate parameters remain in the model but not are not displayed in the figure. Path coefficients without parentheses are for ADHD sample; path coefficients in parentheses are for non-ADHD comparison sample.

${ }^{\dagger} p<.10 .{ }^{*} p<.05 .{ }^{* *} p<.01 . * * * p<.001$

Fig. 2 Cross-lagged Path Model Examining Bidirectional Effects between Coping and Substance Use. Standardized parameter estimates are reported. Non-significant and covariate parameters remain in the model but not are not displayed in the figure. Path coefficients

United States (May/June), as well as during the summer (July/August), and fall (October/November) 2020. Overall, our findings suggest mixed patterns of adjustment such that the pandemic may not have incurred uniformly maladaptive responses. Adolescents' stress and worry about COVID-19 was highest in spring 2020 and decreased in the summer and fall 2020, whereas mean levels of mental health symptoms and substance use were fairly stable throughout the COVID19 timepoints. It is possible the pandemic had a positive impact in these domains for some adolescents by buffering against increased risk for mental health and substance use patterns typically observed in adolescence. Adolescents with ADHD experienced greater mental health symptoms and more frequent substance use, whereas adolescents without ADHD experienced greater stress about the pandemic and COVID-19-related worry.

Positive coping strategies were found to be a buffer for all four of the domains of adjustment examined. Initial levels of adolescent stress, worries about COVID-19, and mental health symptoms predicted increases in coping behaviors and use of routines, which in turn predicted decreased mental health symptoms, worry, and stress, only for adolescents with ADHD. Results highlight coping behaviors and routines were especially critical for individuals with ADHD during spring, summer, and fall 2020. These findings have important clinical implications for promoting adjustment and preventing negative mental health outcomes for this atrisk clinical population during this pandemic and for future chronic stressors.

Our findings that adolescents with ADHD engaged in less adaptive coping behaviors and routines throughout the without parentheses are for ADHD sample; path coefficients in parentheses are for non-ADHD comparison sample. ${ }^{\dagger} p<.10 .{ }^{*} p<.05$. $* * p<.01 . * * * p<.001$

COVID-19 pandemic extend prior research suggesting that youth with ADHD on average engage with more maladaptive and less flexible coping behaviors (e.g. Babb et al., 2010), and that adolescents with ADHD engaged in less routines during the COVID-19 stay-at-home orders (Becker et al., 2020). To date only one study has examined the role of routines in the outcomes of adolescents with ADHD during the COVID-19 pandemic; specifically, Becker and colleagues (2020) linked fewer routines to more remote learning difficulties. Similarly, only two studies have examined coping/ emotion regulation behaviors among youth with ADHD during the COVID-19 pandemic (Breaux et al., 2021a; McFayden et al., 2021). None of these prior studies simultaneously examined coping and routines as predictors of adjustment during the pandemic, and none examined specific coping behaviors (i.e. looking at adaptive coping and emotion regulation abilities as composites). Further, the present study is the first to examine substance use among youth with ADHD during the COVID-19 pandemic. Although similar rates of substance use were reported among adolescents with or without ADHD prior to COVID $(d=0.13)$, adolescents with ADHD reported higher levels of substance use than their peers without ADHD in the spring and summer during COVID-19 $(d s=0.22-0.35)$. This is consistent with findings that ADHD symptoms are associated with increased smoking, alcohol, and marijuana use during periods of increased risk or stress during adolescence (e.g. transition to high school or college; Dvorsky \& Langberg, 2016; Vitulano et al., 2014). Together, the findings from our study and prior research suggest that adolescents with ADHD are particularly at-risk for poor adjustment to chronic stressors, such 
as the COVID-19 pandemic, and efforts to promote adaptive coping and consistent routines could promote resilience in these at-risk youth.

Compared to their peers without ADHD, adolescents with ADHD reported fewer worries about being infected or their health being influenced by COVID-19. This decreased concern or worry among adolescents with ADHD is in stark contrast to emerging evidence indicating that adults with ADHD are more likely to contract COVID-19 and, subsequently, to be at risk for more severe symptoms and outcomes (Wang et al., 2021). Further individuals with ADHD are also more likely to have limited or poorer access to healthcare and to have co-occurring physical conditions (e.g. obesity, sleep disturbances; Avila et al., 2015; Molina \& Pelham, 2014) that are associated with risk for contracting COVID-19 and infection-related outcomes. Moreover, ADHD symptoms have been associated with poorer adherence to COVID-19 preventative measures (e.g. social distancing, mask wearing, personal hygiene; Pollak et al., 2020). ADHD is also associated with increased health risk behaviors such as smoking, substance use, unhealthy food consumption, and driving risks (Barkley, 2015; Molina \& Pelham, 2014). Difficulties in regulating attention and behavior alongside executive function difficulties (i.e. inhibition, working memory, planning) and disruptions in reward processing (i.e. temporal discounting, reward sensitivity) are consistently identified as mechanisms of ADHD-related health risk behavior (Barkley, 2015; Molina \& Pelham, 2014). Decision theory-based studies posit these cognitive systems lead to difficulty in calculating risks, deficit feedback processing, and decreased risk perception, resulting in suboptimal decision making among individuals with ADHD (Pollak et al., 2019). Given this, it is not surprising that adolescents with ADHD may underestimate the gravity of the pandemic and experience less worry about infection and have less concern about the potential health impact of COVID-19.

Adolescents with ADHD also did not experience greater stress about COVID-19 restrictions and changes in familial and social contact compared to adolescents without ADHD. This is somewhat surprising given the potential for pandemic-related shifts (e.g. lack of structure, decreased physical contact with peers, financial hardships) to increase stress, as well as exacerbate ADHD symptoms and associated impairment, among adolescents with ADHD (Bobo et al., 2020; Breaux et al., 2021 a; Sibley et al., 2021). Interestingly, in the present study, increased stress predicted subsequent increases in coping behaviors, such that the presence of stress motivated engagement in healthy coping behaviors but only for adolescents with ADHD. There is growing evidence supporting the assertion that adolescents with ADHD may experience certain positive impacts from COVID-19 (e.g. increased flexibility, time with family, decreased exposure to negative peer interactions at school), despite increased risks and negative impacts (Breaux et al., 2021b). It could be that these and other perceived benefits of the pandemic may play a role in the amount of perceived stress experienced by adolescents with ADHD.

Interestingly, for adolescents with ADHD, higher initial levels of stress, worry, and mental health symptoms were associated with increased use of coping behaviors later during the COVID-19 pandemic timepoints. Despite previous research suggesting that families of youth with ADHD may have more difficulties maintaining routines during the COVID-19 pandemic (Roy et al., 2021), it may be that those with higher levels of stress from COVID-19, worries about COVID-19, and/or mental health symptoms were more motivated to engage in routines or coping behaviors in an effort to combat the negative effects of COVID-19. Specifically, these adolescents with ADHD may have anticipated how the pandemic would adversely affect them, compared to adolescents without mental health concerns, and were more likely to engage in coping behaviors. Other contextual factors coinciding with the timing of assessment not measured in this study (e.g. timing of the school year, exams) may have impacted adolescents' perceived stress. It is also possible that parents of adolescents with ADHD observed negative impacts and facilitated or otherwise supported their adolescent to engage in more coping behaviors. Research in normative adolescent samples examining coping during the pandemic has shown beneficial effects of regular communication with family and friends via video calls, pleasurable and new activities, having a routine (e.g. scheduled times for eating and sleeping), and increased physical activity being identified as useful for combating negative effects and increasing well-being (Pigaiani et al., 2020). Taken together, these results highlight the importance of coping behaviors as a means of managing emotions during COVID-19.

When examining specific domains of coping, we found that adolescents with ADHD who engaged in coping with technology and social coping had decreased mental health symptoms and substance use. Coping with solitary activities was also associated with decreased mental health severity for adolescents with ADHD, whereas social coping was associated with decreased worry about COVID-19 for adolescents without ADHD. Despite potential negative influences of social media on adolescent well-being and mental health, there is research to suggest that social media use has positive influences on sense of belonging and psychosocial wellbeing (Allen et al., 2014). It may be that the social connectedness that comes from playing video games (i.e. technology coping) or talking with friends (i.e. social coping) acts as a buffer against mental health symptoms associated with the pandemic. This is consistent with research by Cauberghe and colleagues (2020), who found adolescents used social media to actively cope with COVID-19 relieved stress and anxiety, which in turn increased feelings of happiness. This 
association may be especially crucial for adolescents with ADHD, who are more likely to experience mental health difficulties associated with the COVID-19 pandemic (Breaux et al., 2021a).

Despite strengths of the current study, including examining risk and protective factors across multiple timepoints during the COVID-19 pandemic, there are several noteworthy limitations. First, we addressed our study aims using a relatively modest sample size of 238 adolescents ( $n=118$ with ADHD), however this sample was not sufficient for simultaneously analyzing these outcomes in a multivariate model, which may capture potential relations among various adjustment outcomes (e.g. mental health impact and substance use). Future research with larger samples is needed to replicate these findings and expand our understanding of adolescents' response to stress and the interrelation between and within coping and adjustment over time. Further, our predominantly White sample of adolescents prevents generalizability of findings to diverse racial/ethnic groups. This is critically important as our study found that adolescents identifying as Black reported greater mental health symptom severity, consistent with research documenting the heightened stress and mental health impact of COVID-19 for Black Americans (e.g. CDC, 2020). Although it would be premature to draw firm conclusions given the small number of Black adolescents in our study, it is important to acknowledge that the higher level of mental health symptom severity among adolescents identifying as Black was during the summer 2020 timepoint which coincides with the substantial social unrest simultaneously occurring in the United States. Future research should incorporate larger, more racially diverse samples to more fully understand the impact of COVID-19 on adolescents' functioning. Additionally, although families who continued to participate across the COVID-19 study timepoints did not differ from the initial sample on demographic characteristics, ADHD symptoms or impairment, it is possible that these families had overall better adjustment and less distress before and during COVID-19. Moreover, this study included adolescents with Combined and Inattentive ADHD Presentations, but was underpowered to examine presentation as a predictor, and future research should consider potential differences presentations of ADHD for coping and adjustment. Of note, the measure used for assessing adolescents' use of various coping strategies (CASPE), was developed during the pandemic and psychometrics for this tool are not yet available. Further, with the exception of parent ratings of adolescents' routines, our measures of mental health, COVID-related worries and stress, substance abuse, and coping behaviors relied on adolescents' self-report, and future work should consider other parentreported domains of adjustment relevant to ADHD such as oppositional behavior, school performance, as well as global functioning. Finally, although our study captured risk and protective factors for the impact of COVID-19 on adolescents across several months of the pandemic, it is unclear how adjustment and coping will unfold as the pandemic draws on and measures are less restrictive, including return to in-person school. An important next step will be testing whether risk and protective factors, particularly adherence to routines and coping behaviors, predict adolescents' long-term mental health outcomes and overall adjustment. In particular, it will be important to examine whether changes in adjustment persist over time, whether mental health symptoms and substance use continue to increase or return to pre-COVID-19 levels as the pandemic continues and ultimately passes. It is also possible that adolescents' substance use patterns were limited by the context of pandemic itself (e.g. stay at home orders, reduced social interactions), which could shift as restrictions end. Determining whether COVID-19-related changes in functioning predict other negative outcomes (e.g. school engagement, social relationships, family functioning) in the future is also important.

This is the first study to our knowledge to prospectively examine changes in adolescent coping and adjustment during the COVID-19 pandemic. The findings shed light on potential differential impacts of the pandemic for adolescents with and without ADHD across multiple adjustment outcomes. The use of positive coping strategies and consistent routines uniquely buffered against risk for maladjustment only in adolescents with ADHD. This study underscores the importance of utilizing routines at the start of a new stressor or abrupt disruption to daily life. These findings have important clinical and public policy relevance for parents and schools, who may wish to prioritize keeping schedules as consistent as possible to promote healthy adjustment. This contrasts with current practices in many communities where repeated cycles of school closures and re-openings have fluctuated schedules based on community positivity rates, in some places multiple times throughout the school year, and disrupting schedules and routines for youth and families. Clinicians should prioritize facilitating routines and enhancing healthy coping behaviors when treating adolescents during the pandemic and future chronic stressors.

Supplementary Information The online version contains supplementary material available at https://doi.org/10.1007/s10802-021-00857-2.

Authors' Contributions SB, JL, RB, and MD contributed to the study conception and design. Material preparation and data collection were performed by SB, RB, and CC. Analyses and interpretations of the data were performed by MD with input from RB and SB. MD led the coordination and drafting of the manuscript. RB, CC, JF, CG, and AS helped to draft the discussion and provided revisions on the manuscript. $\mathrm{SB}, \mathrm{ES}$, and JL provided revisions in the drafting of the manuscript. All authors read and approved the final manuscript. 
Funding This research was supported by a Research Innovation/Pilot award from the Cincinnati Children's Research Foundation (CCRF) and the Virginia Tech COVID-19 Rapid Response Seed Fund, and includes pre-COVID data from and participants previously recruited as part of research supported by award number R305A160126 from the Institute of Education Sciences (IES), U.S. Department of Education. Stephen Becker and Melissa Dvorsky are supported by grants from the National Institute of Mental Health (NIMH; MD: K23MH122839; SB: K23MH108603). The content is solely the responsibility of the authors and does not necessarily represent the official views of the CCRF, Virginia Tech, IES, or NIMH.

Data Availability Data used in the current manuscript is available from the senior author, Stephen Becker, Ph.D., upon request and execution of a data sharing agreement.

Code Availability Mplus syntax used in the growth mixture modeling analyses is available from the first author, Melissa Dvorsky, upon request.

\section{Declarations}

Conflict of interest The authors declare that they have no conflict of interest.

Research Involving Human Participants All procedures performed in studies involving human participants were in accordance with the ethical standards of the institutional and/or national research committee and with the 1964 Helsinki declaration and its later amendments and comparable ethical standards.

Informed Consent Written informed consent was obtained from the parents, and verbal or written (ages 11 and up) was obtained from the adolescents.

\section{References}

Allen, K. A., Ryan, T., Gray, D., McInerney, D., \& Waters, L. (2014). Social media use and social connectedness in adolescents: The positives and the potential pitfalls. Educational and Developmental Psychologist, 31(1), 18-31.

Avila, C., Holloway, A. C., Hahn, M. K., Morrison, K., Restivo, M., Anglin, R., \& Taylor, V. (2015). An overview of links between obesity and mental health. Current Obesity Reports, 4, 303-310.

Babb, K., Levine, L., \& Arseneault, J. (2010). Shifting gears: Coping flexibility in children with and without ADHD. International Journal of Behavioral Development, 34(1), 10-23.

Barkley, R. A. (2015). Attention-deficit/hyperactivity disorder: A handbook for diagnosis and treatment. Guilford.

Becker, S. P., Breaux, R., Cusick, C., Dvorsky, M. R., Marsh, N., Sciberras, E., \& Langberg, J. M. (2020). Remote learning during COVID-19: Examining school practices, service continuation, and difficulties for adolescents with and without ADHD. Journal of Adolescent Health, 67, 769-777.

Becker, S. P., Langberg, J. M., Eadeh, H., Isaacson, P., \& Bourchtein, E. (2019). Sleep and daytime sleepiness in adolescents with and without ADHD: Differences across ratings, daily diary, and actigraphy. Journal of Child Psychology \& Psychiatry, 60(9), 1021-1031.

Bobo, E., Lin, L., Acquaviva, E., Caci, H., Franc, N., Gamon, L.,... \& Purper-Ouakil, D. (2020). How do children and adolescents with
Attention Deficit Hyperactivity Disorder (ADHD) experience lockdown during the COVID-19 outbreak? Encephale, 46(3S), S85-S92.

Breaux, R., Dvorsky, M. R., \& Becker, S. P. (2021b). ADHD in COVID-19: Risk, resilience, and the rapid transition to telehealth. ADHD Report, 29, 1-10.

Breaux, R., Dvorsky, M. R., Marsh, N., Green, C. D., Cash, A., Shroff, D., Buchen, N., Langberg, J. M., \& Becker, S. P. (2021). Prospective impact of COVID-19 on mental health functioning in adolescents with and without ADHD: Protective role of emotion regulation abilities. Journal of Child Psychology \& Psychiatry. Advanced Online. https://doi.org/10.1111/jcpp.13382

Cauberghe, V., Van Wesenbeeck, I., De Jans, S., Hudders, L., \& Ponnet, K. (2020). How adolescents use social media to cope with feelings of loneliness and anxiety during COVID-19 lockdown. Cyberpsychology, Behavior, and Social Networking., 24(4), 250-257.

Centers for Disease Control and Prevention. (2020). Mental Health Household Pulse Survey - COVID-19. Available from: https:// www.cdc.gov/nchs/covid19/pulse/mental-health.htm. Published June 17, 2020 [last accessed 3 March 2021]

DeJong, C. A., Verhagen, J., Pols, R., Verbrugge, C., \& Baldacchino, A. (2020). Psychological impact of the acute COVID-19 period on patients with substance use disorders: We are all in this together. Basic and Clinical Neuroscience, 11(2), 207.

Dumas, T., Ellis, W., \& Litt, D. (2020). What does adolescent substance use look like during the COVID-19 pandemic? Examining changes in frequency, social contexts, and pandemic-related predictors. Journal of Adolescent Health, 67(3), 354-361.

Dvorsky, M. R., Breaux, R., \& Becker, S. P. (2020). Finding ordinary magic in extraordinary times: Child and adolescent resilience during the COVID-19 pandemic. European Child \& Adolescent Psychiatry. https://doi.org/10.1007/s00787-020-01583-8

Dvorsky, M. R., \& Langberg, J. M. (2016). A review of factors that promote resilience in youth with ADHD and ADHD symptoms. Clinical Child and Family Psychology Review, 19(4), 368-391. https://doi.org/10.1007/s10567-016-0216-z

Dvorsky, M. R., Langberg, J. M., Evans, S. W., \& Becker, S. P. (2016). The protective effects of social factors on the academic functioning of adolescents with ADHD. Journal of Clinical Child \& Adolescent Psychology, 47(5), 713-726. https://doi.org/10.1080/ 15374416.2016 .1138406$.

Edwards, S., Rudaizky, D., Toner, M., \& Chen, W. (2021). A Qualitative Study of Factors Perceived by Parents as Promoting Personal Recovery in Children and Young People with ADHD. Advances in Neurodevelopmental Disorders, 5(1), 102-115.

Ellis, W. E., Dumas, T., \& Forbes, L. (2020). Physically isolated but socially connected: Psychological adjustment and stress among adolescents during the initial COVID-19 crisis. Canadian Journal of Behavioural Science, 52(3), 177-187.

Hawke, L. D., Barbic, S., Voineskos, A., Szatmari, P.,... \& Henderson, J. (2020). Impacts of COVID-19 on youth mental health, substance use, and well-being: A rapid survey of clinical and community samples. The Canadian Journal of Psychiatry, 65(10), 701-709.

Ladouceur C. D. COVID-19 Adolescent Symptom and Psychological Experience Questionnaire (CASPE). Pittsburgh, PA: Author; 2020.

Langberg, J. M., Breaux, R. P., Cusick, C. N., Green, C. D., Smith, Z. R., Molitor, S. J., \& Becker, S. P. (2019). Intraindividual variability of sleep/wake patterns in adolescents with and without ADHD. Journal of Child Psychology \& Psychiatry, 60, 1219-1229.

Lee, S. S., Humphreys, K., Flory, K., Liu, R., \& Glass, K. (2011). Prospective association of childhood ADHD and substance use and abuse/dependence: A meta-analytic review. Clinical Psychology Review, 31(3), 328-341. 
Magson, N. R., Freeman, J., Rapee, R., Richardson, C., Oar, E., \& Fardouly, J. (2020). Risk and protective factors for prospective changes in adolescent mental health during the COVID-19 pandemic. Journal of Youth and Adolescence. https://doi.org/10.1007/ s10964-020-01332-9

McClelland, G. H., \& Judd, C. M. (1993). Statistical difficulties of detecting interactions and moderator effects. Psychological Bulletin, 114(2), 376-390.

McFayden, T. C., Breaux, R., Bertollo, J. R., Cummings, K., \& Ollendick, T. H. (2021). COVID-19 remote learning experiences of youth with neurodevelopmental disorders in rural Appalachia. Journal of Rural Mental Health. https://doi.org/10.1037/rmh0000171

Molina, B. S., \& Pelham, W. E., Jr. (2014). Attention-deficit/hyperactivity disorder and risk of substance use disorder: Developmental considerations, potential pathways, and opportunities for research. Annual Review of Clinical Psychology, 10, 607-639.

Moran, M. D. (2003). Arguments for rejecting the sequential Bonferroni in ecological studies. Oikos, 100(2), 403-405.

Muthen, L. K., \& Muthen, B. O. (2020). Mplus. Muthen \& Muthen.

Nikolaidis A, Paksarian D, Alexander L, et al. The Coronavirus Health and Impact Survey (CRISIS) reveals reproducible correlates of pandemic-related mood states across the Atlantic. medRxiv. 2020:2020.2008.2024.20181123.

Paus, T., Keshavan, M., \& Giedd, J. (2008). Why do many psychiatric disorders emerge during adolescence? Nature Reviews Neuroscience, 9, 947-957.

Pigaiani, Y., Zoccante, L., Zocca, A., Arzenton, A., Menegolli, M.,... \& Colizzi, M. (2020). Adolescent Lifestyle Behaviors, Coping Strategies and Subjective Wellbeing during the COVID-19 Pandemic: An Online Student Survey. Healthcare, 8(4), 472.

Piscitello, J., Cummins, R., Kelley, M., \& Meyer, K. (2019). Development and Initial Validation of the Adolescent Routines Questionnaire: Parent and Self-Report. Journal of Psychopathology and Behavioral Assessment, 41(2), 208-220.

Pollak, Y., Dayan, H., Shoham, R., \& Berger, I. (2020). Predictors of adherence to public health instructions during the COVID-19 pandemic. Psychiatry and Clinical Neurosciences. https://doi.org/ 10.1101/2020.04.24.20076620

Pollak, Y., Dekkers, T. J., Shoham, R., \& Huizenga, H. (2019). Risktaking behavior in Attention Deficit/Hyperactivity Disorder (ADHD): A review of potential underlying mechanisms and of interventions. Current Psychiatry Reports, 21(5), 33.

Prime, H., Wade, M., \& Browne, D. T. (2020). Risk and resilience in family well-being during the COVID-19 pandemic. American Psychologist, 75(5), 631-643.

Racine, N., Cooke, J., Eirich, R., Korczak, D., McArthur, B., \& Madigan, S. (2020). Child and adolescent mental illness during COVID-19: A rapid review. Psychiatry Research. https://doi.org/ 10.1016/j.psychres.2020.113307

Ren, H., He, X., Bian, X., Shang, X., \& Liu, J. (2021). The protective roles of exercise and maintenance of daily living routines for Chinese adolescents during the COVID-19 quarantine period. Journal of Adolescent Health, 68(1), 35-42.

Roy, A., Breaux, R., Sciberras, E., Patel, P., Ferrara, E., Shroff, D.,... \& Becker, S.P. (2021). A Preliminary examination of key strategies, challenges, and benefits of remote learning expressed by parents during the COVID-19 pandemic. School Psychology. https://doi. org/10.1037/spq0000465

Sarvey, D., \& Welsh, J. W. (2021). Adolescent substance use: Challenges and opportunities related to COVID-19. Journal of Substance Abuse Treatment, 122, 108212.

Satorra, A., \& Bentler, P. M. (2001). A scaled difference chi-square test statistic for moment structure analysis. Psychometrika, 66(4), 507-514.

Sciberras, E., Patel, P., Stokes, M., Coghill, D., Middeldorp, C.,... \& Westrupp, E. (2020). Physical health, media use, and mental health in children and adolescents with ADHD during the COVID-19 pandemic in Australia. Journal of Attention Disorders. https://doi.org/10.1177/1087054720978549

Sibley, M. H., Ortiz, M., Gaias, L. M., Reyes, R., Joshi, M., Alexander, D., \& Graziano, P. (2021). Top problems of adolescents and young adults with ADHD during the COVID-19 pandemic. Journal of Psychiatric Research, 136, 190-197.

Silk, J., Scott, L., Hutchinson, E., Lu, C., Sequeira, S., McKone, K.,...\& Ladouceur, C. (2021). Storm clouds and silver linings: impacts of COVID-19 and daily emotional health in adolescent girls. PsyArXiv. Retrieved June 15, 2021, from:https://psyarxiv.com/ hmsj8/

Tang, S., Xiang, M., Cheung, T., \& Xiang, Y. (2021). Mental health and its correlates among children and adolescents during COVID19 school closure: The importance of parent-child discussion. Journal of Affective Disorders, 279, 353-360.

Vitulano, M. L., Fite, P. J., Hopko, D. R., Lochman, J., Wells, K., \& Asif, I. (2014). Evaluation of underlying mechanisms in the link between childhood ADHD symptoms and risk for early initiation of substance use. Psychology of Addictive Behaviors, 28(3), 816-827.

Wang, J., \& Wang, X. (2012). Structural equation modeling: Methods and applications. Wiley.

Wang, Q., Xu, W., \& Volkow, N. (2021). Increased risk of COVID-19 infection and mortality in people with mental disorders: Analysis from electronic health records in the United States. World Psychiatry, 20, 124-130.

Zhang, J., Shuai, L., Yu, H., Wang, Z., Qiu, M., Lu, L.,... \& Chen, R. (2020). Acute stress, behavioural symptoms and mood states among school-age children with attention-deficit/hyperactive disorder during COVID-19. Asian Journal of Psychiatry, 51, 102077.

Publisher's Note Springer Nature remains neutral with regard to jurisdictional claims in published maps and institutional affiliations. 